\title{
Fluorescent-Based Methods for Gene Knockdown and Functional Cardiac Imaging in Zebrafish
}

\author{
Noriko Umemoto $\cdot$ Yuhei Nishimura $\cdot$ Yasuhito Shimada $\cdot$ Yukiko Yamanaka \\ Seiya Kishi · Saki Ito $\cdot$ Kana Okamori $\cdot$ Yuuki Nakamura $\cdot$ Junya Kuroyanagi · \\ Zi Zhang $\cdot$ Liqing Zang $\cdot$ Zhipeng Wang $\cdot$ Norihiro Nishimura $\cdot$ Toshio Tanaka
}

Published online: 15 May 2013

(c) The Author(s) 2013. This article is published with open access at Springerlink.com

\begin{abstract}
A notable advantage of zebrafish as a model organism is the ease of gene knockdown using morpholino antisense oligonucleotide (MO). However, zebrafish morphants injected with MO for a target protein often show heterogeneous phenotypes, despite controlling the injection volume of the MO solution in all embryos. We developed a method for estimating the quantity of MO injected into each living morphant, based on the co-injection of a control MO labeled with the fluorophore lissamine. By applying
\end{abstract}

Electronic supplementary material The online version of this article (doi:10.1007/s12033-013-9664-6) contains supplementary material, which is available to authorized users.

N. Umemoto · Y. Nishimura · Y. Shimada · Y. Yamanaka ·

S. Kishi - S. Ito - K. Okamori · Y. Nakamura - J. Kuroyanagi ·

Z. Zhang $\cdot$ L. Zang $\cdot$ Z. Wang $\cdot$ T. Tanaka $(\triangle)$

Department of Molecular and Cellular Pharmacology,

Pharmacogenomics and Pharmacoinformatics, Mie University

Graduate School of Medicine, 2-174 Edobashi, Tsu,

Mie 514-8507, Japan

e-mail: tanaka@doc.medic.mie-u.ac.jp

Y. Nishimura $\cdot$ Y. Shimada $\cdot$ T. Tanaka

Mie University Medical Zebrafish Research Center,

2-174 Edobashi, Tsu, Mie 514-8507, Japan

Y. Nishimura $\cdot$ Y. Shimada $\cdot$ T. Tanaka

Department of Bioinformatics, Mie University Life Science

Research Center, 2-174 Edobashi, Tsu, Mie 514-8507, Japan

Y. Nishimura $\cdot$ Y. Shimada $\cdot$ T. Tanaka

Department of Omics Medicine, Mie University Industrial

Technology Innovation Institute, 2-174 Edobashi, Tsu,

Mie 514-8507, Japan

L. Zang $\cdot$ N. Nishimura

Department of Translational Medical Science, Mie University

Graduate School of Medicine, 2-174 Edobashi, Tsu,

Mie 514-8507, Japan this method for knockdown of cardiac troponin $\mathrm{T}$ (tnnt2a) in zebrafish, we could efficiently select the partial tnnt $2 a$ depleted zebrafish with a decreased heart rate and impairment of cardiac contraction. To investigate cardiac impairment of the tnnt $2 a$ morphant, we performed fluorescent cardiac imaging using Bodipy-ceramide. Cardiac image analysis showed moderate reduction of tnnt $2 a$ impaired diastolic distensibility and decreased contraction and relaxation velocities. To the best of our knowledge, this is the first report to analyze the role of tnnt $2 a$ in cardiac function in tnnt $2 a$-depleted living animals. Our combinatorial approach can be applied for analyzing the molecular function of any protein associated with human cardiac diseases.

Keywords Zebrafish - Gene knockdown - Morpholino · Lissamine - Cardiac function - Bodipy-ceramide - Cardiac troponin $\mathrm{T} \cdot$ Cardiomyopathy $\cdot$ Gene dosage effect $\cdot$ Human disease model

\section{Introduction}

Gene targeting technology, such as gene knockout $(\mathrm{KO})$ and gene knockdown, is used in the targeted analysis of specific gene function in human disease. When downregulation, or reduced activity, of a gene product is inferred to be associated with the cause and progress of a disease, a KO animal for that gene may be developed to evaluate phenotypic similarity to the human disease. However, a particular difficulty arises when the homozygous $\mathrm{KO}$ results in embryonic lethality. To overcome the lethality of the homozygous KO, the phenotype of the heterozygous KO animal can be evaluated. Unfortunately, a $50 \%$ reduction in the expression of a targeted gene in heterozygous mutants rarely results in a 


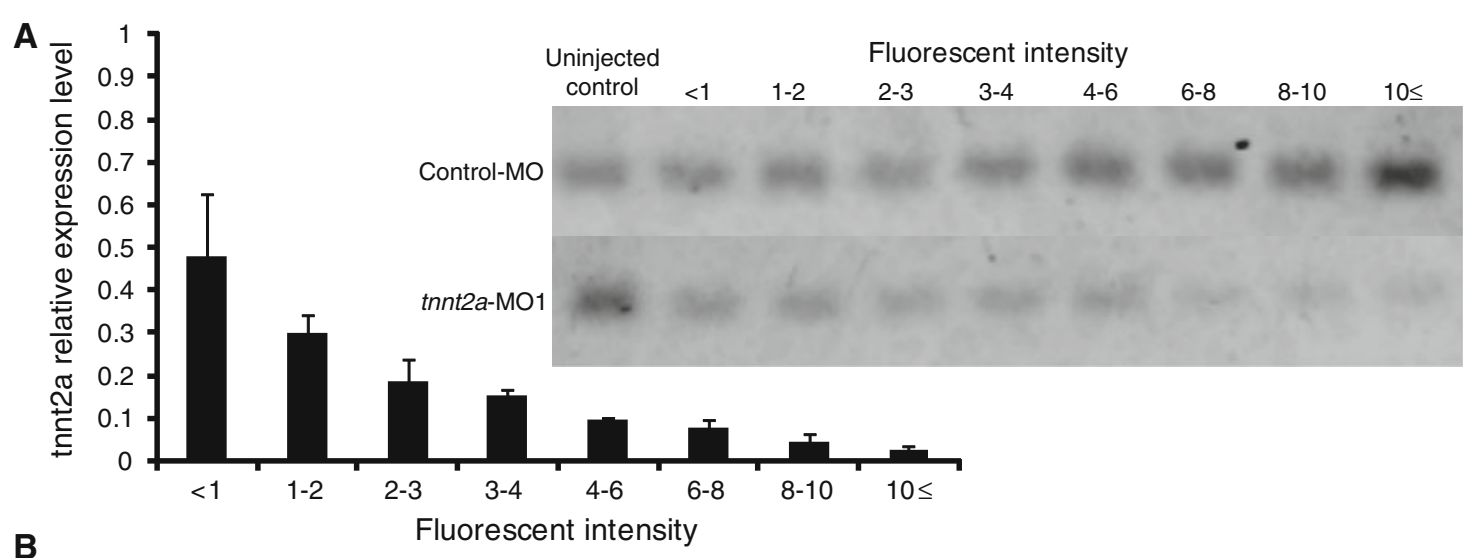

\section{No phenotype}

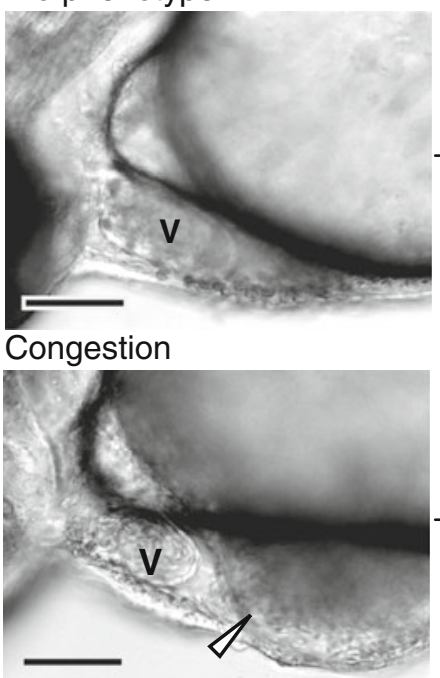

No cardiac output
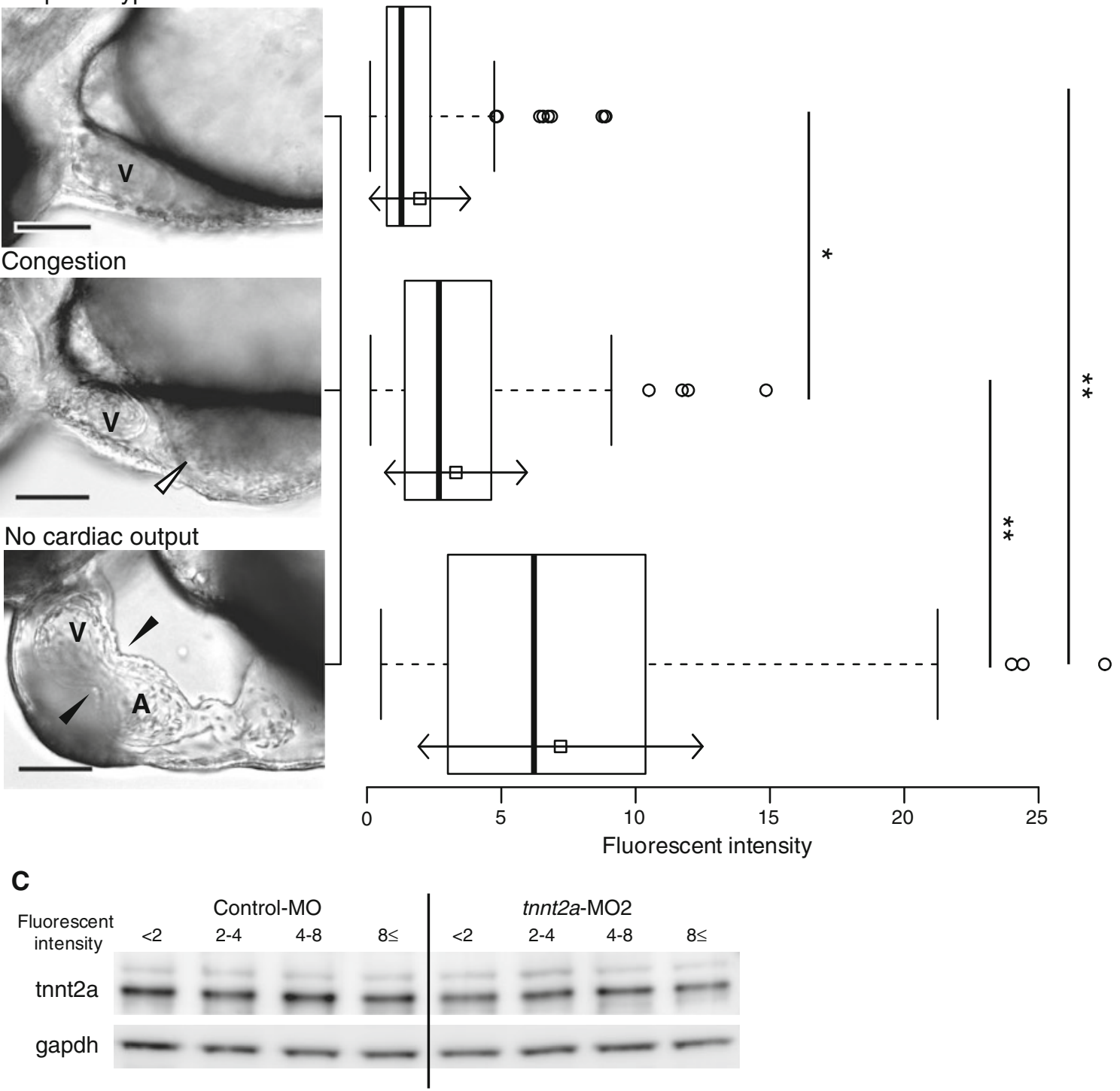

detectable phenotype $[1,2]$. In contrast, little is known regarding phenotypes resulting from a 70 to $80 \%$ reduction in target gene expression [1,2]. This intermediate range may correspond to the variation associated with point mutations or reduced expression levels of alleles of human disease genes $[1,2]$. For example, mutations of cardiac troponin $\mathrm{T}$ (TNNT2) are responsible for $15 \%$ of all cases of familial hypertrophic cardiomyopathy [3]. Troponin is a complex 
4Fig. 1 Expression level of tnnt $2 a$ and the cardiac phenotypes of zebrafish injected with tnnt $2 a-\mathrm{MO}$ are correlated with the FI of LisMO in the embryo. a The expression level of cardiac troponin $\mathrm{T}$ (tnnt2a) was dependent on the fluorescent intensity (FI) from co-injected control antisense oligonucleotides (MO) labeled with the fluorophore lissamine (Lis-MO) in tnnt $2 a$ morphants classified by several ranges of the FI. b Zebrafish injected with $1 \mu \mathrm{M}$ tnnt $2 a-\mathrm{MO} 2$ showed three different cardiac phenotypes: no phenotype; congestion (white arrowhead shows accumulated blood in the sinus venosus); and no cardiac output (black arrowhead shows an abnormal atrioventricular valve). The FI of Lis-MO in zebrafish was significantly different among the three phenotypic groups $(* p<0.05$ and $* * p<0.01$, Bonferroni test). These relative expressions are normalized ratios of tnnt 2 a/gapdh to the control with a FI value less than 1 . The white squares and arrows in the boxplot are the mean value and \pm SD of each group, respectively (no phenotype, $n=112$; congestion, $n=153$; no cardiac output, $n=183$ ). c Western blotting was performed to validate the effect of tnnt $2 a-\mathrm{MO} 2$. The zebrafish embryos injected with $1 \mu \mathrm{M}$ control-MO and tnnt $2 a-\mathrm{MO} 2$ were classified into four groups based on the FI from co-injected Lis-MO: (i) $\mathrm{FI}<2$, (ii) $\mathrm{FI} \geq 2$ and $<4$, (iii) $\mathrm{FI} \geq 4$ and $<8$, and (iv) $\mathrm{FI} \geq 8$. We performed an immunoblotting assay to quantify expression levels of tnnt2a protein in each group $(n=10-17)$. Scale bar $100 \mu \mathrm{m}$. $A$ atrium; $V$ ventricle

composed of three distinct gene products, troponin $\mathrm{C}(\mathrm{TnC})$, troponin I ( TnI), and troponin $\mathrm{T}(\mathrm{TnT})$ in skeletal and cardiac muscles. Homozygous Tnnt $2 \mathrm{KO}$ mice embryos show severe cardiac malformation without a heartbeat and die at approximately embryonic day 10 (E10) [4, 5]. In contrast, Tnnt2 heterozygous KO mice do not show any significant impairment in cardiac function $[4,5]$, making it difficult to analyze the gene dosage effect of Tnnt2 in a beating heart using either homozygous or heterozygous $\mathrm{KO}$ mice. Therefore, a method is required to generate animal models exhibiting intermediate phenotypes in which expression of the gene of interest is precisely reduced [6].

Zebrafish is an attractive model organism for investigation of cardiovascular disease because of its genetic tractability, external fertilization, early optical transparency, and ability to survive without a functional cardiovascular system during development [7]. In particular, the two major advantages of zebrafish embryos are simple gene manipulation using morpholino antisense oligonucleotides (MOs) and live imaging of tissue highlighted by a fluorescent dye or expressed fluorescent proteins [8-10]. Knockdown of any target protein is readily achieved with an MO [9, 11-14]. The expression of the targeted protein is reduced by MOs in a dose-dependent manner [11, 15]. However, one of the difficulties in experiments using MOs is that the quantity of MO injected can slightly vary among embryos, reflecting the severity of the phenotype in the morphants.

To manipulate a targeted gene dosage effect, we improved this method by injecting the MO-targeted certain gene into each embryo by co-injection of control $\mathrm{MO}$ labeled with the fluorophore lissamine (Lis-MO). The control MO has no target in the zebrafish genome. In the current study, we determined the fluorescence intensity (FI) from Lis-MO in each morphant injected with a mixture of the targeted MO and Lis-MO, and determined the relative expression levels of mRNA for the target gene in each morphant. Although previous reports have shown that tnnt $2 a$ morphants with no cardiac contraction completely inhibit translation of tnnt $2 \mathrm{a}$ protein by injecting a high concentration of tnnt $2 a-\mathrm{MO}[16,17]$, we were able to develop tnnt $2 a$ morphants with a homogeneous intermediate phenotype that is in between homozygous and heterozygous Tnnt $2 \mathrm{KO}$ models by applying our method. In addition, to characterize ventricular impairment in the tnnt $2 a$ morphants with impaired cardiac function, we also performed cardiac assessment of zebrafish to measure the performance of the inner-ventricular wall using a commercially available fluorescent dye, Bodipy-ceramide [18]. In the present study, functional cardiac imaging of zebrafish stained with Bodipy-ceramide was able to detect both systolic and diastolic dysfunction in the heart, albeit a fluorescent protein-labeled heart.

\section{Materials and Methods}

The investigation conformed to the ethical guidelines established by the Institutional Animal Care and Use Committee of Mie University.

\section{Zebrafish Breeding}

Care and breeding of zebrafish were as described previously [19]. Poorly pigmented nacre mutants of zebrafish [20] were used in this study because of their greater transparency. Homozygous nacre mutants lack melanophores throughout development, but have increased numbers of iridophores and have a mutation in a zebrafish gene, microphthalmia-associated transcription factor a (mitfa). Zebrafish embryos were obtained by natural spawning of the nacre line.

\section{Injection of MOs}

All MOs used in this study were purchased from Gene Tools LLC (Philomath, OR, USA) and prepared and injected as previously described [14]. As a negative control, embryos were injected with a control MO (control-MO), which theoretically has no target in the zebrafish genome. The sequence of the control-MO was 5'-GAGACTTCATCT TACCTCATATTTC- $3^{\prime}$. We also used control-MO with lissamine attached to the $3^{\prime}$ end (Lis-MO) to monitor the quantity of $\mathrm{MO}$ for the gene of interest that was present in the embryos after microinjection. We used two MOs to knockdown tnnt $2 a$. The first MO, tnnt2a-MO1, was used to block 
the splicing of exon 3 of tnnt $2 a$, and to analyze the correlation between the FI of Lis-MO and the knockdown level of tnnt2a in the embryos. The sequence of this MO was $5^{\prime}$ AACATAAGACTTAACCCTCCTGCTCC- $3^{\prime}$. The second MO (tnnt2a-MO2) was used to block translation of tnnt $2 a$ mRNA and to analyze the correlation between the FI of Lis$\mathrm{MO}$ and the severity of the cardiac phenotype. The sequence of this MO was reported in a previous study [17]. We injected MOs into fertilized eggs at the 1-4-cell stage using a FemtoJet microinjector (Eppendorf, Hamburg, Germany) with constant injection pressure and injection time. The settings for the FemtoJet microinjector were $50 \mathrm{hPa}$ for compensation pressure, $150 \mathrm{hPa}$ for injection pressure, and $0.5 \mathrm{~s}$ for injection time. For knockdown of tnnt $2 a$, we injected a solution of $20 \mu \mathrm{M}$ tnnt $2 a-\mathrm{MO} 1$ or $1 \mu \mathrm{M}$ tnnt $2 a$ $\mathrm{MO} 2+50 \mu \mathrm{M}$ Lis-MO into embryos. The negative control was 20 or $1 \mu \mathrm{M}$ control-MO $+50 \mu \mathrm{M}$ Lis-MO. The embryos were then allowed to develop in egg water.

Measurement of the FI of Lissamine Conjugated to Control MO Co-injected into Zebrafish Embryos

After anesthesia using either $0.016 \%$ tricaine (SigmaAldrich, St. Louis, MO, USA) or 100 ppm 2-phenoxyethanol (Wako, Osaka, Japan), zebrafish embryos injected with MOs were placed into a 96-well plate with egg water at $60 \mathrm{~h}$ post-fertilization (hpf). The FI from lissamine (excitation/ emission: 575/593 nm) of each zebrafish embryo was measured using a fluorescent microplate reader (Varioskan, Thermo Fisher Scientific, Waltham, MA, USA) (Fig. 1a, b).

\section{Quantitative PCR (qPCR) Analysis}

The morphants at $60 \mathrm{hpf}$ were each placed into a microcentrifuge tube and treated with RNAlater (Ambion, Austin TX, USA) after measurement of the FI from Lis-MO. Total RNA from morphants was isolated using the RNAqueous ${ }^{\circledR}$-Micro Kit (Ambion). Total RNA was used to generate cDNAs using the iScript Select cDNA Synthesis Kit (Bio-Rad, Hercules, CA, USA). Quantitative PCR for analyzing the quantity of tnnt2a and gapdh was carried out using an ABI Prism 7300 (Life Technologies, Carlsbad, CA, USA) with SYBR Green Real-time PCR Master Mix Plus (Toyobo, Osaka, Japan). The thermal cycling conditions comprised an initial step at $95{ }^{\circ} \mathrm{C}$ for $1 \mathrm{~min}$, followed by 40 cycles of $95^{\circ} \mathrm{C}$ for $15 \mathrm{~s}$, $60{ }^{\circ} \mathrm{C}$ for $15 \mathrm{~s}$, and $72{ }^{\circ} \mathrm{C}$ for $45 \mathrm{~s}$. Data were normalized by the quantity of glyceraldehyde-3-phosphate dehydrogenase (gapdh). This allowed us to account for any variability in the initial template concentration, as well as the conversion efficiency of the reverse transcription reaction. The primers used in this study were: tnnt $2 a-\mathrm{F}, 5^{\prime}$-CAACGAAGAAGTG GAAGAGTACGAG - $3^{\prime}$; tnnt $2 a$-R, $5^{\prime}$-TTCTCCATCGTGT TCCTGAGTG - $3^{\prime}$; gapdh-F, $5^{\prime}$-TTCTCACAAACGAGGA
CACAA- ${ }^{\prime}$, and $g a p d h$-R, $5^{\prime}$-CAAGGTCAATGAATGGG TCA- $3^{\prime}$. For visualization, the quantitative PCR product was loaded on a $2.5 \%$ agarose gel and the gel was electrophoresed in a Mupid-2 (Cosmo Bio. Co. Ltd., Tokyo, Japan) electrophoresis tank at $50 \mathrm{~V}$ per $\mathrm{cm}$ for $80 \mathrm{~min}$ using $0.5 \times$ TBE buffer.

Injection of tnnt $2 a$ mRNA for Phenotypic Rescue of the tnnt2a Morphant

The zebrafish tnnt $2 a$ coding region (amino acids 1-282) obtained from ZGC clone 7234566 (Open Biosystems, Huntsville, AL, USA) was subcloned into the EcoRI and $X b a \mathrm{I}$ sites of the plasmid pCS2P+ (Addgene, Cambridge, MA, USA). The sequence of the construct was confirmed by automated DNA sequencing. The sequence containing full-length cDNA for tnnt $2 a$ was amplified by PCR using the following primer pairs: $5^{\prime}$-TGAGATACCTACAGCG TGAGC-3' ${ }^{\prime}$ and $5^{\prime}$-CCGAGATAGGGTTGAGTGTTG- $3^{\prime}$. The PCR product was used as the template for RNA synthesis. Messenger RNA for tnnt $2 a$ was synthesized using the mMessage mMachine transcription kit for SP6 RNA polymerase (Ambion). For the rescue experiment, a solution containing $0.13 \mathrm{ng} / \mathrm{nl}$ of tnnt $2 a$ mRNA (approximately $0.39 \mu \mathrm{M})$ and $1 \mu \mathrm{M}$ of tnnt $2 a-\mathrm{MO} 2$ was injected into 1-4cell stage embryos, which were then raised in egg water at $28^{\circ} \mathrm{C}$ until 60 hpf.

\section{Protein Isolation and Western Blot Analysis}

Embryos injected with MO were dechorionated at $60 \mathrm{hpf}$ and were lysed in Tissue Protein Extraction Reagent (T-PER, Thermo Fisher Scientific) containing protease inhibitor cocktail (Thermo Fisher Scientific). The Pierce ${ }^{\circledR} \mathrm{BCA}^{\mathrm{TM}}$ protein assay kit (Thermo Fisher Scientific) was used to determine protein concentrations. The protein extract $(25 \mu \mathrm{g})$ was loaded onto 4-12\% sodium dodecyl sulfate polyacrylamide gels, electrophoresed, and transferred to polyvinylidene fluoride (PVDF) membranes using NuPAGE and iBlot Systems (Invitrogen, Carlsbad, CA, USA). After electroblotting, membranes were blocked in PVDF Blocking Reagent for Can Get Signal ${ }^{\circledR}$ (Toyobo) at room temperature or $37{ }^{\circ} \mathrm{C}$ for $1 \mathrm{~h}$ and probed with primary antibodies at $4{ }^{\circ} \mathrm{C}$ overnight as follows: Ab-1 (Thermo Fisher Scientific) against tnnt2a using a $500 \times$ dilution in Can Get Signal ${ }^{\circledR}$ solution 1 (Toyobo); and ab36840 (Abcam, Cambridge, MA, USA) against gapdh, using a $3000 \times$ dilution in Can Get Signal ${ }^{\circledR}$ solution 1 . The membranes were washed thrice in phosphatebuffered saline (PBS) supplemented with $0.05 \%$ Tween 20 (PBS-T) and incubated with horseradish peroxidase-conjugated secondary antibody (Abcam) diluted in Can Get Signal $^{\circledR}$ solution 2 for $1 \mathrm{~h}$ at room temperature. The 
membranes were washed again thrice in PBS-T and developed with ImmunoStar ${ }^{\circledR}$ LD (Wako).

Fluorescent Cardiac Imaging of Zebrafish Using Bodipy-ceramide

Zebrafish embryos were dechorionated and immersed for $3-5 \mathrm{~h}$ in egg water containing $0.2 \mu \mathrm{M}$ of Bodipy-ceramide (BODIPY $^{\circledR}$ FL C5-ceramide, Invitrogen). The stained embryos were placed on a coverslip and embedded in $3 \%$ methylcellulose solution. Embryos were arranged with the anterior to the left of the field and ventral surface down. In this position, the ventricle was clearly visible using an inverted florescence microscope (Axiovert 200M, Carl Zeiss, Oberkochen, Germany). Finally, the embryo was covered with $2 \%$ ultralow gelling agarose (Agarose Type VII, A4018, Sigma-Aldrich) to fix its position. A coverslip was placed on the stage of the microscope (Thermo plate, Tokai Hit, Shizuoka, Japan) and maintained at $28^{\circ} \mathrm{C}$. Time-lapse images of the zebrafish ventricle were recorded at approximately 20 frames per second using AxioVision software (Rel. 4.6, Carl Zeiss) and a digital camera (AxioCam, Carl Zeiss). The imaging duration was $5 \mathrm{~s}$ using a GFP filter and $15 \mathrm{~s}$ for bright field.

Cardiac Image Analysis Using Bodipy-ceramide Staining

We used MBF ImageJ (NIH, Bethesda, MD, USA) [21] to process the original records for the measurement of cardiac
Fig. 2 Fluorescent cardiac imaging of zebrafish ventricles using Bodipy-ceramide. ad The images display representative individual timelapse images from bright-field and Bodipy-ceramide stained embryos. In fluorescence images at ventricular diastole (c) and systole (d), the boundaries between the cardiac wall and the lumen can be more clearly distinguished than in bright-field images at ventricular diastole (a) and systole (b). Scale bar $50 \mu \mathrm{m}$. e To generate M-mode images, the intersection of the horizontal (long) axis and the vertical (short) axis lines of the "orthogonal views" was positioned at the "center of mass" (arrowhead). M-mode images were then generated, with each slice representing the "pseudo-linescan" of a singlepixel-wide line along these horizontal and vertical lines. $\mathrm{M}$-mode images were created from serial time-lapse images of the moving ventricle with Bodipy-ceramide staining. $L$ long axis, $S$ short axis, $t$ time. f The mean ventricular wall velocities during systole (mVWVs) or diastole $(\mathrm{mVWVd})$ were calculated as the $a-b$ slope and $b-c$ slope, respectively
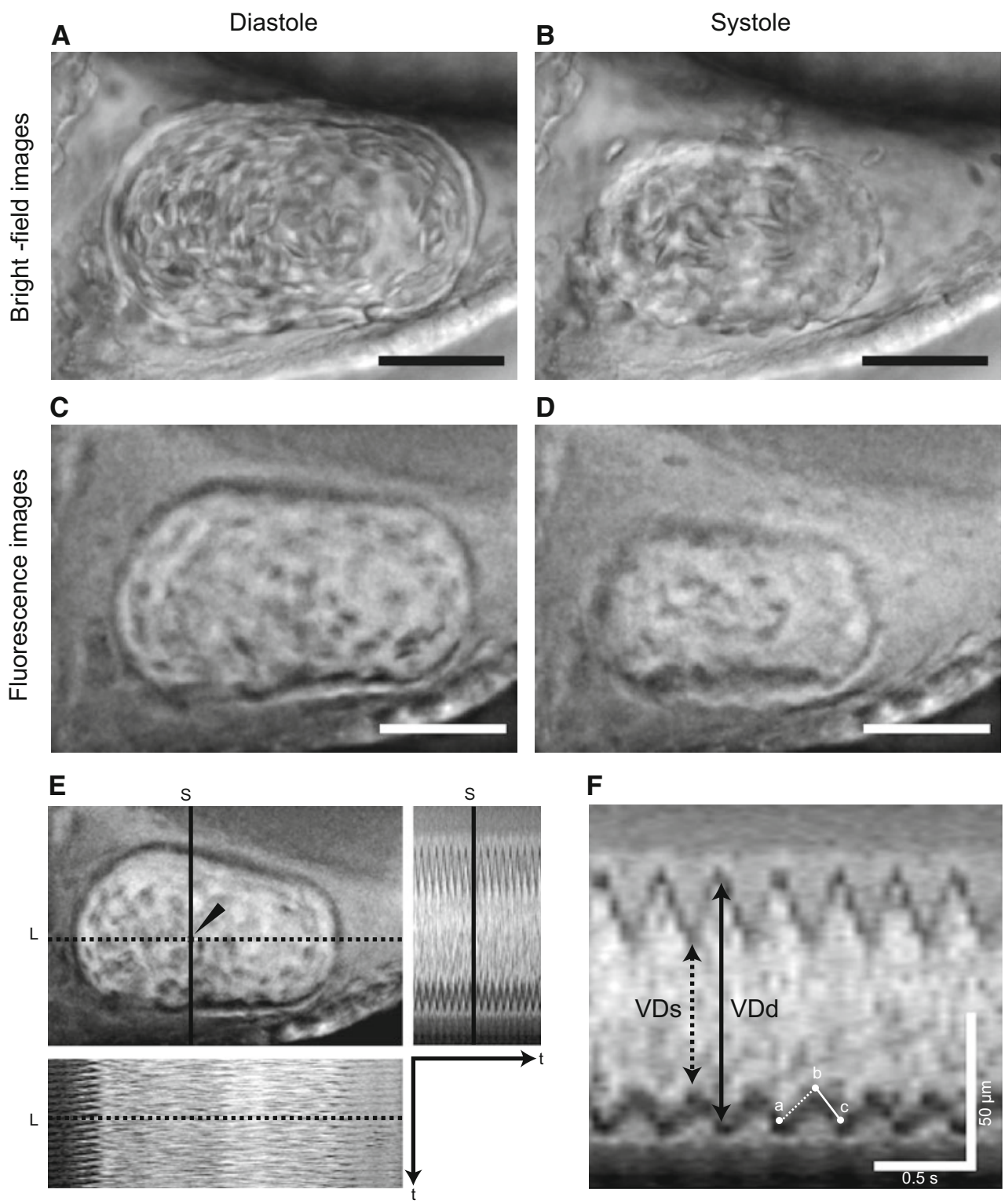
performance using Bodipy-ceramide cardiac imaging. The "iterative deconvolution" plugin was used to enhance the contrast between the plasma and the ventricular wall. The inner perimeter of the ventricle in the end systolic frame was outlined using a mouse or graphic tablet, from which its "center of mass", and subsequently, the bestfitting ellipse, were calculated [22]. The coordinates of the center of mass were memorized using the region of interest manager tool in MBF ImageJ. To make M-mode images in the long or short axes of the ventricle, the intersection of horizontal and vertical lines of the "orthogonal view" was positioned at the center of mass. M-mode images were then generated, with each slice representing a "pseudo-linescan" a single-pixel-wide along these horizontal and vertical lines. The ventricular end systolic dimension (VDs) and the ventricular end diastolic dimension (VDd) were measured from the M-mode images. VDs and VDd were obtained at maximal inward and outward excursions, respectively, of the ventricular wall in the M-mode images. We measured VDs and VDd along the short axis (short VDs, short VDd) and the long axis (long VDs, long VDd). For each determination, five diastoles and systoles were analyzed. The end diastolic volume (EDV) and end systolic volume (ESV) were calculated using the formula for a prolate spheroid $\left(4 \times a \times b^{2} / 3\right)$ [22]. The stroke volume (SV) was obtained by subtracting the ESV from the EDV. The ejection fraction (EF) was calculated as SV/EDV and the percentage fractional shortening $(\% \mathrm{FS})$ was calculated from the formula: 100(short VDd - short VDs)/short VDd. We also obtained coordinate data from a total of 11 points, representing the maximal inward (6 points) and outward (5 points) excursions of the ventral ventricular wall in the M-mode images. Mean ventricular wall velocities during systole (mVWVs) or diastole ( $\mathrm{mVWVd}$ ) were averages of five slopes at systole or diastole, respectively (Fig. 2f). We also counted the number of heartbeats for $15 \mathrm{~s}$, as described previously [13].

\section{The Time-Volume Curve (TVC) and Its Differentiation Curve}

The inner perimeter of the ventricle in each frame was outlined using the freehand selection tool in MBF image J. The lengths of the long and short axes of the ventricle in each frame were calculated from the perimeter of each ventricle using the best-fitting ellipse algorithm. Therefore, the ventricular volumes in each frame were calculated based on these axes data and entered into a volume curve differentiation program (VCDiff, Fujifilm RI Pharma, Tokyo, Japan) [23]. In this program, Fourier curve fitting was performed using five harmonics, and cardiac parameters were automatically calculated from the TVC and its differentiation curve (Fig. 4a) as follows: $\mathrm{EDV}(\mathrm{pl})=\mathrm{V} 1$; $\mathrm{ESV}(\mathrm{pl})=\mathrm{V} 2 ; \mathrm{EF}(\%)=100(\mathrm{~V} 1-\mathrm{V} 2) / \mathrm{V} 1 ;$ first third $\mathrm{EF}$ $(1 / 3 \mathrm{EF}, \%)=100(\mathrm{~V} 1-\mathrm{V} 3) / \mathrm{V} 1$; first third filling fraction $(1 / 3 \mathrm{FF}, \%)=100(\mathrm{~V} 4-\mathrm{V} 2) /(\mathrm{V} 1-\mathrm{V} 2)$; time to peak ejection $(\mathrm{TPE}, \mathrm{ms})=\mathrm{TD} 1$; time to peak filling $(\mathrm{TPF}, \mathrm{ms})=$ $\mathrm{TD} 2$; first third ejection rate $(1 / 3 \mathrm{ER}, \mathrm{pl} / \mathrm{ms})=-\mathrm{D} 3 \times$ $1,000 / \mathrm{V} 1$; peak ejection rate $(\mathrm{PER}, \mathrm{pl} / \mathrm{ms})=-\mathrm{D} 1 \times 1,000 /$ $\mathrm{V} 1$; first third filling rate $(1 / 3 \mathrm{FR}, \mathrm{pl} / \mathrm{ms})=\mathrm{D} 4 \times 1,000 /$ $\mathrm{T} \times \mathrm{V} 1$; and peak filling rate $(\mathrm{PFR}, \mathrm{pl} / \mathrm{ms})=\mathrm{D} 2 \times 1,000 / \mathrm{V} 1$.

\section{Statistical Analysis}

Statistical analysis was performed using SAS version 9.1 (SAS Institute, Cary, NC, USA) and R version 2.15.1 [24]. Spearman's correlation coefficient by rank test was used to analyze the correlation between the expression level of tnnt $2 a$ and the FI from lissamine in each tnnt $2 a$ morphant. Significant differences for the mean FIs and cardiac parameters among the three groups were identified using the Bonferroni multiple comparisons test. Determination of the statistical difference for cardiac parameters was determined using the two-sided Student's $t$ test for comparison of two groups. The Chi square test was used to analyze the relationship between cardiac phenotype and the level of tnnt $2 a$ mRNA. Differences with $p<0.05$ were considered significant.

\section{Results}

The Knockdown Level of tnnt $2 a$ in Zebrafish Injected with tnnt $2 a$-MO Can be Estimated by the FI of Co-injected Lissamine-MO

To estimate the amount of MO present in each embryo after microinjection, a mixture of an MO targeted to the gene of interest and Lis-MO was injected into embryos at the 1-4-cell stage. We performed co-injection of Lis-MO to measure the knockdown efficiency of tnnt $2 a$ in zebrafish embryos. We designed tnnt $2 a-\mathrm{MO} 1$ to knockdown tnnt $2 a$ by skipping exon 3 and quantified the expression level of tnnt $2 a$ in the morphants classified by several ranges of FI level by qPCR analysis. The FI from lissamine was assumed to co-vary with the quantity of MO injected into the eggs. As shown in Fig. 1a, the expression level of tnnt2a was dependent on the FI from co-injected Lis-MO in tnnt2a morphants (Fig. 1a). This observation suggested that the FI from Lis-MO co-injected with any MO-targeted gene of interest could be used to indirectly estimate the knockdown efficiency of the gene. 
The Severity of Cardiac Impairment in tnnt $2 a$ Morphants is Dependent on the FI of Co-injected Lissamine-MO

We injected a mixture of tnnt $2 a-\mathrm{MO} 2$ to knockdown tnnt $2 a$ by blocking translation and Lis-MO at $50 \mu \mathrm{M}$. The tnnt $2 a$ morphants showed three different phenotypes at $60 \mathrm{hpf}$ : (i) no phenotype; (ii) congestion; and (iii) no cardiac output (Fig. 1b). To analyze the correlation between the severity of cardiac impairment and the FI from co-injected Lis-MO, we measured the FI from Lis-MO and classified the morphants into these cardiac phenotypes (Fig. 1b, boxplots). The Bonferroni multiple comparisons test showed that the mean FI values were significantly different among the three cardiac phenotypes (no phenotype vs. congestion, $p<0.05$; no phenotype vs. no cardiac output and congestion vs. no cardiac output, $p<0.01)$. The severity of cardiac impairment in the morphants injected with tnnt $2 a$-MO1, which was mentioned above, was also dependent on the FI from coinjected Lis-MO (Fig. S1). Representative movies of the morphant and the control are shown in Supplemental movies 1-6. The severity of cardiac impairment was dependent on the FI, which was also correlated with the expression levels of tnnt $2 a$ in the morphants. Therefore, we conclude that co-injection of Lis-MO permits the selection of morphants with expression levels reduced by MO.

The Cardiac Phenotypes of Zebrafish Injected with tnnt $2 a-\mathrm{MO}$ are Attributed to Knockdown of tnnt2a Protein

To confirm whether the cardiac phenotypes in zebrafish injected with tnnt $2 a-\mathrm{MO} 2$ were caused by knockdown of tnnt $2 a$, we quantified the level of tnnt 2 a protein expression in zebrafish embryos using immunoblotting. As shown in Fig. S2A, the major band corresponding to a splicing variant of tnnt $2 a$ [25] was reduced in zebrafish injected with tnnt $2 a-\mathrm{MO} 2$ at $10 \mu \mathrm{M}$. Morphants injected with tnnt $2 a-\mathrm{MO} 2$ at $10 \mu \mathrm{M}$ showed a weak or non-beating heart with severe abnormal morphology, which was correlated with the FI from co-injected Lis-MO (Fig. S2B-M). Representative movies of the morphant and the control are shown in Supplemental movies 7-12. Subsequently, we also detected a decrease in tnnt $2 \mathrm{a}$ protein in the tnnt $2 \mathrm{a}$ morphants, which showed a FI greater than 8.0, when injected with tnnt $2 a-\mathrm{MO} 2$ at $1 \mu \mathrm{M}$. Moreover, we observed a slight reduction in the expression level of tnnt 2 a protein in morphants with an FI up to 8.0 from Lis-MO. This finding is probably because of the insufficient sensitivity of immunoblotting to accurately quantify tnnt 2 a protein levels in zebrafish (Fig. 1C, Fig. S3).

To confirm the specificity of the knockdown effect of tnnt $2 a-\mathrm{MO} 2$ on the cardiac phenotypes, we co-injected
Table 1 Phenotypic rescue of tnnt $2 a$ morphants by co-injection of tnnt $2 a$ mRNA

\begin{tabular}{llcc}
\hline & \multicolumn{2}{c}{ Cardiac phenotypes } & Total \\
\cline { 2 - 3 } & No phenotype & Congestion & \\
\hline tnnt2a-MO2 & 2 & 13 & 15 \\
tnnt2a-MO2 + tnnt2a mRNA & 16 & 4 & 20 \\
Total & 18 & 17 & 35 \\
\hline
\end{tabular}

tnnt $2 a$ mRNA with tnnt $2 a-\mathrm{MO} 2$ into zebrafish eggs. At 60 hpf, 13 of 15 zebrafish embryos injected with tnnt $2 a$-MO2 without tnnt $2 a$ mRNA showed congestion in the sinus venosus (Table 1). In contrast, only four of 20 zebrafish embryos injected with tnnt $2 a-\mathrm{MO} 2$ with tnnt $2 a$ mRNA showed congestion (Table 1). Cardiac impairment was significantly improved by co-injection of tnnt $2 a$ mRNA $(p<0.05$, Chi square test), which suggested that the cardiac phenotype induced by injection of tnnt $2 a-\mathrm{MO} 2$ could be attributed to the knockdown of tnnt $2 a$.

Development of Fluorescent Cardiac Image Analysis Using Bodipy-ceramide Staining to Assess Cardiac Performance in Zebrafish

To analyze the cardiac function of tnnt $2 a$ in zebrafish with respect to cardiac output, it is important to establish methods to measure the internal ventricular dimensions. For this purpose, the boundary between the ventricular wall and the lumen must be clearly defined. However, it is difficult to detect the boundary in bright-field images because the cardiac wall of zebrafish embryos is transparent (Fig. 2a, b). To visualize the cardiac lumen, we used Bodipy-ceramide, which is used to visualize blood flow in the zebrafish heart for the analysis of the intracardiac fluid force [26] and to detect abnormal valvulogenesis during heart development [27]. Zebrafish embryos stained with Bodipy-ceramide showed a clear boundary between the ventricular wall and the lumen (Fig. 2c, d). After Bodipy-ceramide staining, the beating ventricle was recorded using fluorescence microscopy. M-mode images were created from fluorescence timelapse images of the ventricle (Fig. 2e), and from these images, we measured cardiac parameters, including VDs, VDd, mVWVs, and mVWVd (Fig. 2f). Our data showed that fluorescent cardiac imaging with Bodipy-ceramide could be used to assess cardiac function in zebrafish.

The reliability of cardiac imaging using Bodipy-ceramide for measurement of cardiac function was tested on the developing heart of zebrafish. The sequence of cardiac development is as follows (Fig. S4A): heart tube formation at $22 \mathrm{hpf}$, looping at $36 \mathrm{hpf}$, valve formation at $48 \mathrm{hpf}$, and adult configuration at $120 \mathrm{hpf}$ [28-30]. We assessed cardiac 

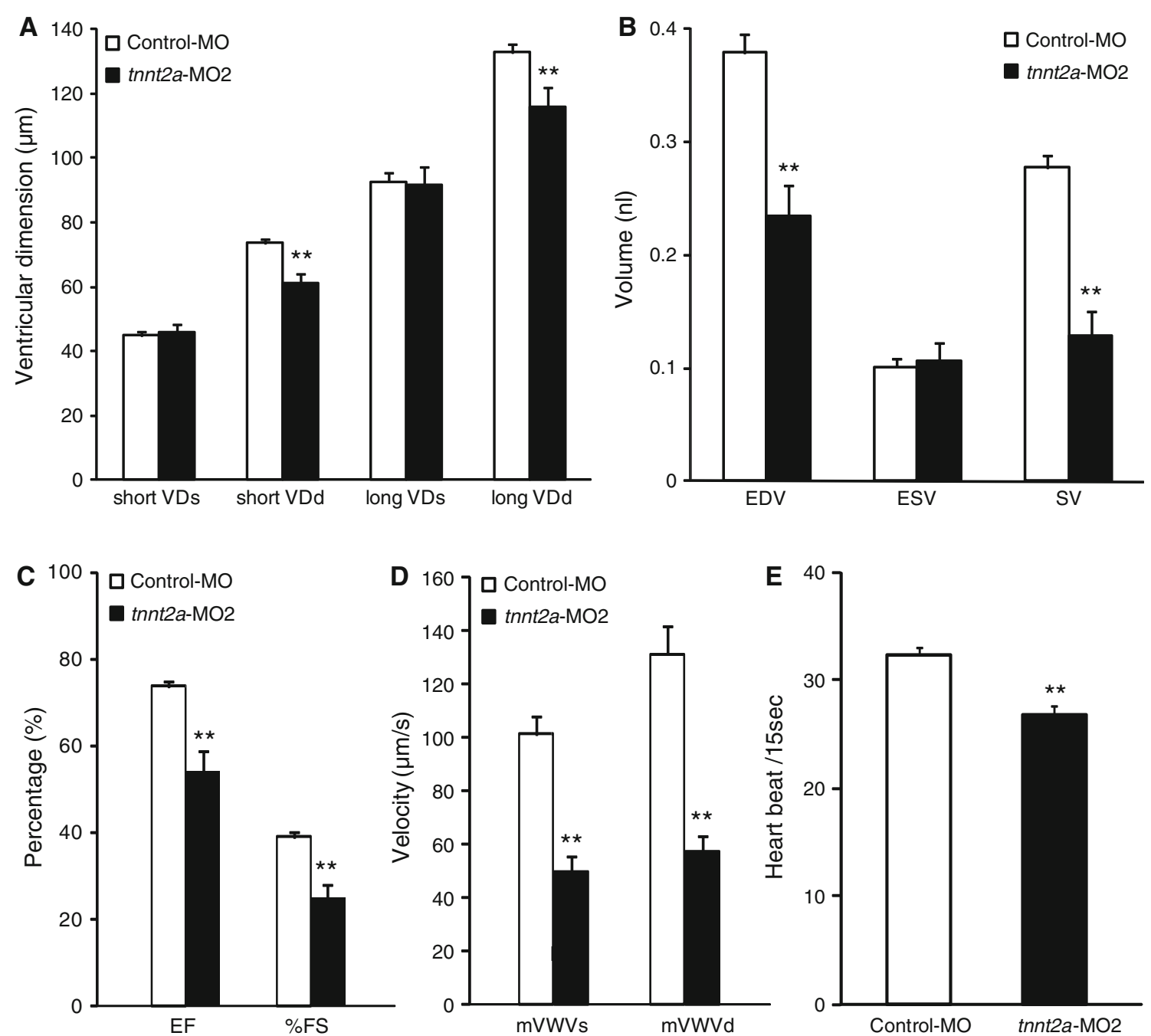

Fig. 3 Impairment of diastolic distensibility and ventricular wall velocities during systole and diastole in zebrafish by knockdown of tnnt $2 a$. In the control group, zebrafish were injected with control MO $(1 \mu \mathrm{M})$ and Lis-MO $(50 \mu \mathrm{M})$. In the tnnt $2 a$ knockdown group, zebrafish were injected with tnnt $2 a-\mathrm{MO} 2(1 \mu \mathrm{M})$ and Lis-MO $(50 \mu \mathrm{M})$. We selected zebrafish embryos, which showed FI values between 2 and 5 in the control group at $60 \mathrm{~h}$ post-fertilization (hpf), and compared them with tnnt $2 a$ morphants with blood congestion and FI values from 2 to 5 in the tnnt $2 a$ knockdown group at $60 \mathrm{hpf}$. a-d Cardiac parameters were measured from M-mode images of

function at 60, 72, and $96 \mathrm{hpf}$ because, at these stages, zebrafish have been reported to exhibit marked changes in cardiac performance [31]. In our study, mean VDs and VDd were decreased along with both short and long axes between 60 and 72 hpf ( $p<0.05$, Fig. S4B), and between 60 and 96 hpf ( $p<0.05$, Fig. S4B). Consequently, ventricular volumes (ESV, EDV) and SV were significantly lower at 72 and 96 hpf than those at 60 hpf ( $p<0.05$, Fig. S4C), while EF and \%FS were increased from 60 to $96 \mathrm{hpf}$ ( $p<0.05$, Fig. S4D). The 72 and $96 \mathrm{hpf}$ values for cardiac parameters (ventricular dimension, ventricular volume, EF and \% FS) were all significantly different from the $60 \mathrm{hpf}$ values, except ventricles in groups of zebrafish injected with either control-MO or tnnt $2 a-\mathrm{MO} 2$ at $60 \mathrm{hpf}$ : a The ventricular end systolic dimension (VDs) and the ventricular end diastolic dimension (VDd) of both short and long axes; $\mathbf{b}$ the end diastolic volume (EDV) and end systolic volume (ESV) and stroke volume (SV); c ejection fraction (EF) and fractional shortening (\%FS); and $\mathbf{d} \mathrm{mVWVs}$ and $\mathrm{mVWVd}$. Values are mean $\pm \mathrm{SE}$ (control-MO, $n=24$; tnnt $2 a-\mathrm{MO} 2, n=10$ ). Statistical analysis was performed to examine significant differences from the mean control values $\left({ }^{* *} p<0.01, * p<0.05\right.$, Student's $t$ test)

for the long VDs and EF values at $72 \mathrm{hpf}$ (Fig. S4B-E, $p<0.05$, Bonferroni tests). Contraction velocity, mVWVs, was significantly higher at 72 and 96 hpf compared with that at $60 \mathrm{hpf}$, and corresponding (non-significant) changes were observed in mean mVWVd ( $p<0.05$, Fig. S4E).

Knockdown of tnnt $2 a$ Impairs Diastolic Distensibility and Cardiac Wall Velocity

We focused on tnnt $2 a$ morphants with congestion in the sinus venosus for further analysis of cardiac function because congestion might reflect a circulatory abnormality 
induced by reducing tnnt $2 a$ expression. We chose tnnt $2 a$ morphants with FI values between 2 and 5 because the percentage of tnnt $2 a$ morphants with congestion in the sinus venosus was highest in this group. Fluorescent cardiac image analysis was applied to this group. We found that, in zebrafish injected with tnnt $2 a-\mathrm{MO} 2$, VDd, but not VDs, was significantly reduced compared with that in control-MO injections ( $p<0.01$, Fig. 3a). Accordingly, EDV and SV, but not ESV, were significantly reduced compared with control values ( $p<0.01$, Fig. 3b). EF and $\%$ FS were unchanged in zebrafish injected with tnnt $2 a$ MO2 (Fig. 3c). These results suggested that knockdown of tnnt $2 a$ expression selectively impaired diastolic distensibility, which led to congestion in the sinus venosus. From the M-mode images, we also measured ventricular wall velocities during systole and diastole. Both mVWVs and $\mathrm{mVWVd}$ were significantly reduced in zebrafish injected with tnnt $2 a-\mathrm{MO} 2$ compared with those in controls $(p<0.01$, Fig. 3d). These results suggested that the knockdown of tnnt $2 a$ expression delayed ventricular wall motion equally during contraction and relaxation.

In addition, we performed TVC analysis using fluorescent cardiac images (Fig. 4a, b). In zebrafish injected with tnnt $2 a-\mathrm{MO} 2, \mathrm{EDV}$ and EF were significantly decreased compared with those in controls ( $p<0.05$, Fig. 4c, d), consistent with observations using the M-mode method. PER and PFR were significantly decreased in zebrafish injected with tnnt $2 a-\mathrm{MO} 2$ compared with those in zebrafish injected with control-MO (Fig. 4f, $p<0.05$ ), but TPE and TPF were not significantly different between the groups (Fig. 4e). Ventricular wall velocities (mVWVs and $\mathrm{mVWVd}$ ) and peak rates (PER and PFR) during systole and diastole showed velocities of contraction and relaxation that were reflected by systolic function and diastolic function, respectively. These results suggested that velocities of contraction and relaxation were lower in tnnt $2 a$ morphant ventricles than those in control zebrafish. Consistent with these findings, the heart rate of tnnt $2 a$ morphants was significantly decreased compared with that in control zebrafish ( $p<0.01$, Fig. 3e).

\section{Discussion}

Co-injection of a Fluorophore-Labeled MO with the MO for the Gene of Interest is Useful for Classifying Phenotypes Based on the Knockdown Level of the Gene

In practice, the quantity of MO actually injected into embryos can vary to some extent, even when they are injected with nominally similar quantities of MO. The embryos show multiple phenotypes, because the knockdown efficiency of MO depends on the quantity of MO in each morphant [15]. Therefore, it is important to measure the knockdown efficiency of the target protein in the morphants.

To evaluate the knockdown efficiency of MOs, Kamachi et al. [15] generated a modified luciferase assay, which involved two steps: (i) synthesis of the RNA containing $5^{\prime}$-UTR and a short N-terminal coding sequence of the target gene fused with mRNA coding luciferase; and (ii) co-injection of MO for the target and the fused RNA. However, this method is unable to evaluate the knockdown efficiency in living embryos. It is also complex to prepare the fused RNA for each target protein.

In an alternative approach, in the current study, we indirectly estimated the knockdown efficiency in living embryos co-injected with Lis-MO and a MO to knockdown the target protein, and then measured the FI of the Lis-MO in each morphant. When we validated this method with the MO to knockdown tnnt $2 a$, the expression levels of tnnt $2 a$ mRNA in the morphants were negatively correlated with their FI. Therefore, our method has the advantage of being able to generate gene knockdown models showing relatively mild phenotypes caused by reduction of expression of a particular gene. However, a disadvantage of our method is that the knockdown effect of MO gradually disappears over approximately 7 days post-fertilization (dpf). Because the number of nuclei greatly increases during development of zebrafish embryos, the intranuclear concentration of MOs may significantly decrease, thereby reducing their knockdown effect [14]. However, our new method is useful for analysis of the effect of expression levels of a gene-of-interest on biological function, at least during developmental stages. These results confirm that measurement of FI of Lis-MO in living embryos is a convenient and reliable assay to estimate knockdown levels of a target protein.

\section{Cardiac Function of Zebrafish Can be Quantitatively Assessed by Fluorescent Cardiac Imaging Using Bodipy-ceramide}

Zebrafish are a valuable animal model for cardiovascular research because their high transparency allows their beating hearts to be easily observed under the optical microscope. However, measurements of cardiac parameters in zebrafish are subject to inaccuracy because of difficulties in the identification of the boundary between the cardiac wall and the lumen of the heart. In previous reports, transgenic zebrafish were generated that enabled the cardiac wall to be visualized based on the expression of fluorescent proteins in the wall (e.g., $\operatorname{Tg}(\mathrm{cmlc} 2:$ GFP) and SAG4A) [32-34]. Because not all laboratories have access to these transgenic zebrafish, a novel method to assess cardiac function without these fish lines will be useful for 
A
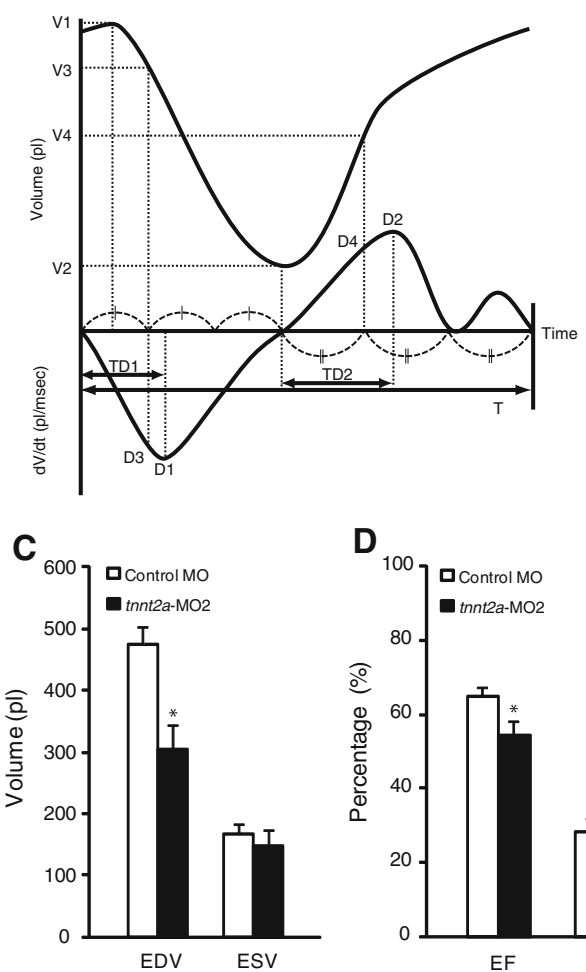

D

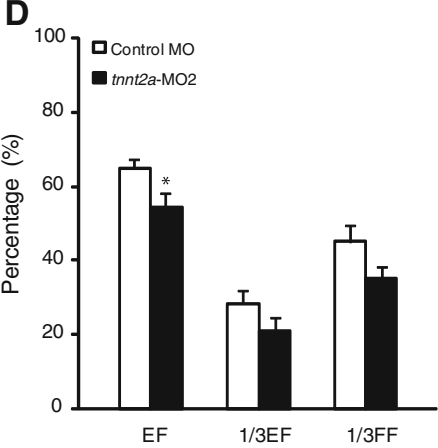

Fig. 4 Time-volume curve (TVC) analysis showing decreased EDV, $\mathrm{EF}$, and peak rates during systole and diastole by knockdown of tnnt $2 a$. We also assessed cardiac parameters of both experimental groups using TVC analysis. a The upper and lower graphs show the TVC and the differentiation curve, respectively. b The TVC and the differentiation curve of control zebrafish are shown (left) alongside those of a tnnt $2 a$ morphant with congestion in the sinus venosus (right). c-f Cardiac function was assessed using TVC analysis based on fluorescent cardiac images using Bodipy-ceramide. The calculated

cardiovascular research by using zebrafish as a model animal. In the current study, we applied fluorescent imaging technology using commercially available Bodipy-ceramide for assessment of cardiac function in zebrafish. Previously, Bodipy-ceramide has been used to visualize blood flow for the analysis of the intracardiac fluid force [26] and to detect abnormal valvulogenesis [27]. In particular, Hove et al. [26] used Bodipy-ceramide to visualize the blood flow pattern inside the heart chambers by labeling blood serum for high-speed fluorescent confocal imaging. Vermot et al. [27] also used Bodipy-ceramide to visualize valve formation and blood flow patterns using high-speed fluorescent confocal imaging. In our study, we showed that fluorescent cardiac imaging with Bodipy-ceramide enhanced the contrast of the boundary between the cardiac wall and the lumen, permitting measurement of many functional parameters, including end systolic dimension, end diastolic dimension, $\mathrm{ESV}, \mathrm{EDV}, \mathrm{EF}, \% \mathrm{FS}$, mVWVs, and mVWVd from M-mode images, and other parameters from TVCs (Fig. 4a). Using fluorescent cardiac
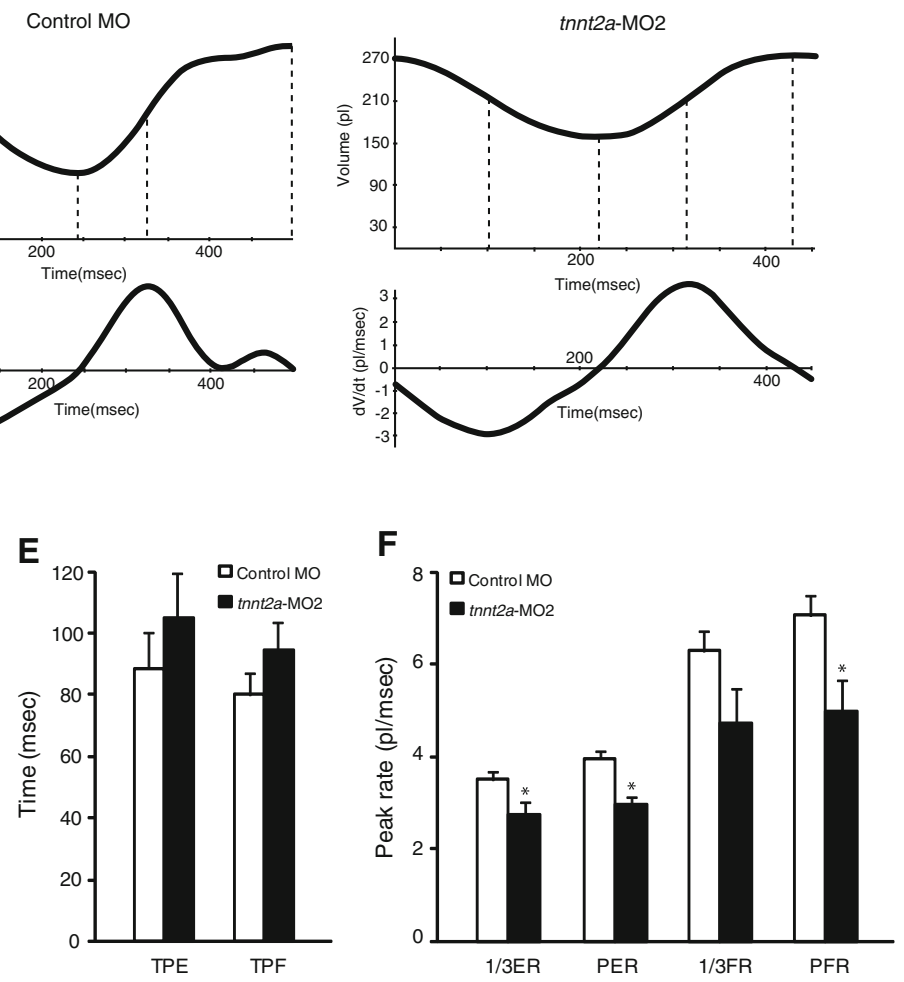

parameters were as follows: c EDV and ESV; d EF, first third EF $(1 / 3 \mathrm{EF})$, and first third filling fraction (1/3FF); $\mathbf{e}$ time to peak ejection (TPE) and time to peak filling (TPF); and $\mathbf{f}$ first third ejection rate (1/3ER), peak ejection rate (PER), first third filling rate (1/3FR) and peak filling rate (PFR). Values are mean $\pm \mathrm{SE}$ (control MO, $n=24$; tnnt $2 a-\mathrm{MO} 2, n=10)$. Statistical analysis was performed to examine significant differences from the mean control values $\left(^{*} p<0.05\right.$, Student's $t$ test)

imaging, we found that intraventricular volume significantly decreased from 60 to $96 \mathrm{hpf}$ during development of the heart. The reduction in SV and the increase in mVWVs at these developmental stages are consistent with previous reports [22, 30, 31]. Additionally, cardiac trabeculae start to form in the ventricle at approximately $72 \mathrm{hpf}$, and the ventricle becomes extensively trabeculated by $5 \mathrm{dpf}$ [35]. Cardiac trabeculation might be responsible for reduction of ventricular volume from 60 to $96 \mathrm{hpf}$. These results suggested that cardiac imaging with Bodipy-ceramide reliably measured cardiac function in zebrafish.

\section{Characterization of Cardiac Function in the tnnt2a Morphant Using Fluorescent-Based Quantitative Assays}

By using the two methods developed in this study, we were able to analyze cardiac function of tnnt $2 a$ morphants showing congestion in the sinus venosus. To the best of our knowledge, this study is the first report to reveal cardiac 
dysfunction induced by depletion of tnnt $2 a$ using a vertebrate with a beating heart.

We found that VDd, but not VDs, of tnnt $2 a$ morphants was significantly shorter than that in control zebrafish. TnT consists of an extended amino-terminal portion, which lies alongside tropomyosin ( $\mathrm{Tm}$ ) on the thin filament, and a globular carboxy-terminal domain that binds to Tm, as well as to TnI and TnC. TnT is involved in distributing the inhibitory effect of the troponin complex, via Tm, to seven actin monomers with which it interacts, in the absence $\mathrm{Ca}^{2+}$. TnT is also involved in removing this inhibitory effect from all seven actin monomers, as well as activating the actomyosin ATPase, in the presence of $\mathrm{Ca}^{2+}$ [36]. Many mutations related to cardiomyopathy have been reported in TNNT2 [3, 36, 37]. In transgenic mice with Tnnt2-R92Q, which show weaker binding to Tm than in wild-type Tnnt2, left ventricular end diastolic volume, but not left ventricular end systolic volume, is significantly reduced compared with that in control mice. Conversely, in transgenic mice with Tnnt2-R141W, which show stronger binding to Tm than in wild-type Tnnt2, left ventricular enddiastolic volume is significantly increased compared with that in control mice [38]. These results suggest that impairment of diastolic distensibility in the tnnt $2 a$ morphant may be caused by decreased binding to Tm.

Our analysis also showed that the ventricular wall velocity for contraction ( $\mathrm{mVWVs}$ and PER) and relaxation (mVWVd and PFR) were significantly decreased in tnnt $2 a$ morphants compared with those of control zebrafish. Interestingly, both maximum shortening and re-lengthening velocity of isolated ventricular cardiomyocytes in Tnnt2R92Q transgenic mice are significantly decreased, which is also consistent with our observations [39]. These results suggest that there may be a common pathophysiological mechanism between knockdown of tnnt $2 a$ and Tnnt2-R92Q. Further studies are required to examine this possibility.

\section{Conclusions}

Our combinatorial approach developed in this study can be applied for analyzing molecular function of proteins associated with human cardiac diseases, especially when the homozygous $\mathrm{KO}$ is lethal and the heterozygous $\mathrm{KO}$ animal shows no significant abnormality.

Acknowledgments We are grateful to Shingo Kato, Masato Yonezawa, and Noriko Ii for helpful discussion regarding cardiac imaging, Takayuki Okamoto for helpful suggestions regarding quantification of expression levels of cardiac troponin $\mathrm{T}$ protein, and Tomomi Yamada for assisting with statistical analyses. We also thank Tomoko Murata, Yumi Yoshikawa, Kimiko Nishiguchi, Chizuru Suzuki-Hirota, Ai Kamakura, and Hiroko Nakayama for their experimental assistance, and Rie Ikeyama, Yoshiko Yoshida, Keiko
Ito, and Yuka Tamura for secretarial assistance. This work was supported by the New Energy and Industrial Technology Development Organization, the Ministry of Education, Culture, Sports, Science and Technology, Mie Prefecture, Japan, and a Young Investigator Award from Mie University Graduate School of Medicine, Japan.

Conflict of interest The authors declare that they have no competing interests.

Open Access This article is distributed under the terms of the Creative Commons Attribution License which permits any use, distribution, and reproduction in any medium, provided the original author(s) and the source are credited.

\section{References}

1. Kleinhammer, A., Deussing, J., Wurst, W., \& Kühn, R. (2011). Conditional RNAi in mice. Methods, 53(2), 142-150.

2. Delic, S., Streif, S., Deussing, J. M., Weber, P., Ueffing, M., Hölter, S. M., et al. (2008). Genetic mouse models for behavioral analysis through transgenic RNAi technology. Genes, Brain, and Behavior, 7(7), 821-830.

3. Knollmann, B. C., \& Potter, J. D. (2001). Altered regulation of cardiac muscle contraction by troponin $\mathrm{T}$ mutations that cause familial hypertrophic cardiomyopathy. Trends in Cardiovascular Medicine, 11(5), 206-212.

4. Nishii, K., Morimoto, S., Minakami, R., Miyano, Y., Hashizume, K., Ohta, M., et al. (2008). Targeted disruption of the cardiac troponin $\mathrm{T}$ gene causes sarcomere disassembly and defects in heartbeat within the early mouse embryo. Developmental Biology, 322(1), 65-73.

5. Ahmad, F., Banerjee, S., Lage, M., Huang, X., Smith, S., Saba, S., et al. (2008). The role of cardiac troponin $T$ quantity and function in cardiac development and dilated cardiomyopathy. PLOS ONE, 3(7), e2642.

6. Lee, S. K., \& Kumar, P. (2009). Conditional RNAi: towards a silent gene therapy. Advanced Drug Delivery Reviews, 61(7-8), 650-664.

7. Tu, S., \& Chi, N. C. (2012). Zebrafish models in cardiac development and congenital heart birth defects. Differentiation, 84(1), 4-16.

8. Oka, T., Nishimura, Y., Zang, L., Hirano, M., Shimada, Y., Wang, Z., et al. (2010). Diet-induced obesity in zebrafish shares common pathophysiological pathways with mammalian obesity. BMC Physiology, 10, 21.

9. Watanabe, K., Nishimura, Y., Oka, T., Nomoto, T., Kon, T., Shintou, T., et al. (2010). In vivo imaging of zebrafish retinal cells using fluorescent coumarin derivatives. BMC Neuroscience, $11,116$.

10. Tainaka, T., Shimada, Y., Kuroyanagi, J., Zang, L., Oka, T., Nishimura, Y., et al. (2011). Transcriptome analysis of anti-fatty liver action by Campari tomato using a zebrafish diet-induced obesity model. Nutrition and Metabolism, 8, 88.

11. Nasevicius, A., \& Ekker, S. (2000). Effective targeted gene 'knockdown' in zebrafish. Nature Genetics, 26(2), 216-220.

12. Tanaka, T., Oka, T., Shimada, Y., Umemoto, N., Kuroyanagi, J., Sakamoto, C., et al. (2008). Pharmacogenomics of cardiovascular pharmacology: Pharmacogenomic network of cardiovascular disease models. J Pharmacol Sci, 107(1), 8-14.

13. Wang, Z., Nishimura, Y., Shimada, Y., Umemoto, N., Hirano, M., Zang, L., et al. (2009). Zebrafish beta-adrenergic receptor 
mRNA expression and control of pigmentation. Gene, 446, $18-27$.

14. Eisen, J., \& Smith, J. (2008). Controlling morpholino experiments: Don't stop making antisense. Development, 135(10), $1735-1743$.

15. Kamachi, Y., Okuda, Y., \& Kondoh, H. (2008). Quantitative assessment of the knockdown efficiency of morpholino antisense oligonucleotides in zebrafish embryos using a luciferase assay. Genesis, 46(1), 1-7.

16. Huang, W., Zhang, R., \& Xu, X. (2009). Myofibrillogenesis in the developing zebrafish heart: A functional study of tnnt2. Developmental Biology, 331(2), 237-249.

17. Sehnert, A. J., Huq, A., Weinstein, B. M., Walker, C., Fishman, M., \& Stainier, D. Y. (2002). Cardiac troponin T is essential in sarcomere assembly and cardiac contractility. Nature Genetics, 31(1), 106-110.

18. Cooper, M., D'Amico, L., \& Henry, C. (1999). Confocal microscopic analysis of morphogenetic movements. Methods in Cell Biology, 59, 179-204.

19. Westerfield, M. (2007). The zebrafish book: A guide for the laboratory use of zebrafish (Danio rerio). Eugene: University of Oregon Press.

20. Lister, J., Robertson, C., Lepage, T., Johnson, S., \& Raible, D. (1999). nacre encodes a zebrafish microphthalmia-related protein that regulates neural-crest-derived pigment cell fate. Development, 126(17), 3757-3767.

21. Collins, T. (2007). ImageJ for microscopy. BioTechniques, 43(1 Suppl), 25-30.

22. Jacob, E., Drexel, M., Schwerte, T., \& Pelster, B. (2002). Influence of hypoxia and of hypoxemia on the development of cardiac activity in zebrafish larvae. American Journal of Physiology: Regulatory, Integrative and Comparative Physiology, 283(4), R911-R917.

23. Nakae, I., Matsuo, S., Tsutamoto, T., Matsumoto, T., Mitsunami, K., \& Horie, M. (2007). Assessment of cardiac function in patients with heart disease by quantitative gated myocardial perfusion SPECT. Annals of Nuclear Medicine, 21(6), 315-323.

24. Gentleman R IR: The R Project for Statistical Computing. R Development Core Team. http://www.R-project.org.

25. Hsiao, C., Tsai, W., Horng, L., \& Tsai, H. (2003). Molecular structure and developmental expression of three muscle-type troponin T genes in zebrafish. Developmental Dynamics, 227(2), 266-279.

26. Hove, J., Köster, R., Forouhar, A., Acevedo-Bolton, G., Fraser, S., \& Gharib, M. (2003). Intracardiac fluid forces are an essential epigenetic factor for embryonic cardiogenesis. Nature, 421(6919), 172-177.

27. Vermot, J., Forouhar, A., Liebling, M., Wu, D., Plummer, D., Gharib, M., et al. (2009). Reversing blood flows act through klf2a to ensure normal valvulogenesis in the developing heart. PLoS Biology, 7(11), e1000246.

28. Bakkers, J. (2011). Zebrafish as a model to study cardiac development and human cardiac disease. Cardiovascular Research, 91(2), 279-288.

29. Glickman, N., \& Yelon, D. (2002). Cardiac development in zebrafish: coordination of form and function. Seminars in Cell \& Developmental Biology, 13(6), 507-513.

30. Denvir, M., Tucker, C., \& Mullins, J. (2008). Systolic and diastolic ventricular function in zebrafish embryos: influence of norepenephrine, MS-222 and temperature. BMC Biotechnology, $8,21$.

31. Malone, M. H., Sciaky, N., Stalheim, L., Hahn, K. M., Linney, E., \& Johnson, G. L. (2007). Laser-scanning velocimetry: A confocal microscopy method for quantitative measurement of cardiovascular performance in zebrafish embryos and larvae. BMC Biotechnology, 7, 40.

32. Kawakami, K., Takeda, H., Kawakami, N., Kobayashi, M., Matsuda, N., \& Mishina, M. (2004). A transposon-mediated gene trap approach identifies developmentally regulated genes in zebrafish. Developmental Cell, 7(1), 133-144.

33. Seguchi, O., Takashima, S., Yamazaki, S., Asakura, M., Asano, Y., Shintani, Y., et al. (2007). A cardiac myosin light chain kinase regulates sarcomere assembly in the vertebrate heart. The Journal of Clinical Investigation, 117(10), 2812-2824.

34. Richardson, P. (1996). Assessment of myocardial damage in dilated cardiomyopathy. European Heart Journal, 17(4), 489-490.

35. Liu, J., Bressan, M., Hassel, D., Huisken, J., Staudt, D., Kikuchi, K., et al. (2010). A dual role for ErbB2 signaling in cardiac trabeculation. Development, 137(22), 3867-3875.

36. Redwood, C. S., Moolman-Smook, J. C., \& Watkins, H. (1999). Properties of mutant contractile proteins that cause hypertrophic cardiomyopathy. Cardiovascular Research, 44(1), 20-36.

37. Gomes, A., Barnes, J., Harada, K., \& Potter, J. (2004). Role of troponin $\mathrm{T}$ in disease. Molecular and Cellular Biochemistry, 263(1-2), 115-129.

38. Lombardi, R., Bell, A., Senthil, V., Sidhu, J., Noseda, M., Roberts, R., et al. (2008). Differential interactions of thin filament proteins in two cardiac troponin $\mathrm{T}$ mouse models of hypertrophic and dilated cardiomyopathies. Cardiovascular Research, 79(1), $109-117$.

39. Tardiff, J. C., Hewett, T. E., Palmer, B. M., Olsson, C., Factor, S. M., Moore, R. L., et al. (1999). Cardiac troponin T mutations result in allele-specific phenotypes in a mouse model for hypertrophic cardiomyopathy. The Journal of Clinical Investigation, 104(4), 469-481. 